University of Nebraska - Lincoln

DigitalCommons@University of Nebraska - Lincoln

Development of House Flies (Diptera: Muscidae) in Sand Containing Varying Amounts of Manure Solids and Moisture

Jerome Hogsette

USDA-ARS, Jerry.Hogsette@ars.usda.gov

Follow this and additional works at: https://digitalcommons.unl.edu/usdaarsfacpub

Part of the Agricultural Science Commons

Hogsette, Jerome, "Development of House Flies (Diptera: Muscidae) in Sand Containing Varying Amounts of Manure Solids and Moisture" (1996). Publications from USDA-ARS / UNL Faculty. 1009.

https://digitalcommons.unl.edu/usdaarsfacpub/1009

This Article is brought to you for free and open access by the U.S. Department of Agriculture: Agricultural Research Service, Lincoln, Nebraska at DigitalCommons@University of Nebraska - Lincoln. It has been accepted for inclusion in Publications from USDA-ARS / UNL Faculty by an authorized administrator of DigitalCommons@University of Nebraska - Lincoln. 


\title{
Development of House Flies (Diptera: Muscidae) in Sand Containing Varying Amounts of Manure Solids and Moisture
}

\author{
JEROME A. HOGSETTE
}

Medical and Veterinary Entomology Research Laboratory, USDA-ARS, P.O. Box 14565, Gainesville, FL 32604

\begin{abstract}
J. Econ. Entomol. 89(4): 940-945 (1996)
ABSTRACT House flies, Musca domestica L., developed in $200 \mathrm{~cm}^{3}$ of coarse sand containing just $1 \mathrm{ml}(0.47 \%)$ of dairy manure solids and $10 \mathrm{ml}(4.74 \%)$ of moisture. At these levels, development was slow (21.5 d from lst instar to adult), adult survival was low (7.5\%), but successful development did occur. At higher manure and moisture levels, rates of development and survival were similar to those reported previously. All soil samples collected from a feedlot dairy contained higher levels of manure solids than the highest level tested in the laboratory. The implications for fly control in soil/manure mixtures and the need for additional studies are discussed.
\end{abstract}

KEY WORDS Musca domestica, larval development, adult survival, nutrient content

THE Literature IS replete with descriptions of materials exploited by house flies, Musca domestica L., for the development of their immature stages (Newstead 1908, Bishopp et al. 1915, James 1947, Sacca 1964). Although a number of organic substances can be utilized, animal manures have been documented as preferred substrates (Hewitt 1914, Mellor 1919, Keiding 1976, Rabari and Patel 1977).

House fly development sites in manure-laden soil have been defined on dairy (Meyer and Shultz 1990) and beef cattle confinement facilities (Skoda et al. 1993). However, no studies have been done to quantify the amount of manure needed in the soil for fly development to occur. Larvae of $\mathrm{Hy}$ drotaea irritans (Fallen) have been found only in pastures in soil substrates (Robinson and Luff 1976), but no subsequent studies have been performed to further define the habitat.

The objective of this study was to quantify the amount of manure solids required for house fly development in sand under varying moisture levels. Levels of moisture and manure solids were varied incrementally to determine whether fly development could be limited by decreasing amounts of manure or increasing amounts of moisture. These studies were inspired by past observations of stable fly, Stomoxys calcitrans (L.), larvae developing 10$15 \mathrm{~cm}$ deep in soil in pastures occupied by horses in south Florida, and house fly larvae developing $15-30 \mathrm{~cm}$ deep in lots containing $\approx 60 \mathrm{~cm}$ of builder's sand and occupied by cattle on north Florida feedlot dairies (unpublished data). With the contimued encroachment of suburban development into traditional agricultural areas and the increased conflict between animal producers and home owners over fly nuisance problems (Thomas and Skoda
1993), it is imperative that animal producers be aware of all areas on their farms capable of producing flies so adequate control strategies can be implemented (Patterson 1981).

\section{Materials and Methods}

House flies from the USDA Gainesville multiresistant colony were used for all tests. Basic rearing techniques were similar to those used by Hogsette (1992), unless otherwise stated.

Treatments were formulated in coarse (30-65 mesh) builder's sand because it closely simulates the type of sand used in confinement lots on many feedlot dairies in Florida. Treatments were based on levels of moisture originating from water alone, manure moisture alone, or from a combination of the two. Within each moisture level, there were 5 water/manure moisture combinations: (1) $100 \%$ moisture from water, (2) $75 \%$ moisture from water and $25 \%$ moisture from manure, (3) $50 \%$ moisture from water and $50 \%$ moisture from manure, (4) $25 \%$ moisture from water and $75 \%$ moisture from manure, and (5) $100 \%$ moisture from manure.

Moisture levels ranged from 10 to $130 \mathrm{ml}$. At each moisture level, moisture (amount, not percentage) was held constant and the accompanying manure solids (nutrients) increased from zero in water/manure moisture combination 1 to a maximum in water/manure moisture combination 5 (Table 1).

Manure used in these studies was $<12$ h old and field-collected from a feedlot dairy milking herd. A portion of the manure was dried to determine moisture content $(81.3 \%)$, and the remainder was placed in air-tight containers and frozen $\left(-17.8^{\circ} \mathrm{C}\right)$ until needed. Treatments were replicated 4 times 
at each level, and each replicate was formulated by mixing $\approx 272.2 \mathrm{~g}\left(200 \mathrm{~cm}^{3}\right)$ of sand with a predetermined amount of manure, water, or both manure and water. Each formulated replicate was then loosely packed into a $240-\mathrm{ml}(10-\mathrm{cm}$ high) clear plastic specimen cup.

Newly hatched house fy larvae were added to each treatment cup using the technique of Hogsette and Washington (1995). House fly females were allowed to oviposit into $\approx 50 \mathrm{ml}$ of conditioned medium (i.e., medium that had already been used for rearing house fly lavae) (Bryant and Hall 1975) for 1-2 h. After $24 \mathrm{~h}$ in the growth chamber, newly hatched larvae were ready for use. This ensured that the large number of larvae hatched from the eggs would be concentrated in a relatively small volume of medium. Medium with larvae was placed in a porcelain tray ( 41 by 25 by $6 \mathrm{~cm}$ high). Larvae were picked from the medium and transferred to the surface of the treatment substrates (50 per cup) with a natural-bristle artist's brush (No. 4) moistened with water.

Cups were covered with muslin cloth secured with rubber bands and held in the growth chamber at $26.7^{\circ} \mathrm{C}$ and $60 \% \mathrm{RH}$. Development time to the pupal stage was recorded. Larvae generally pupated within $5 \mathrm{~cm}$ from the surface of the sand mixture, and pupae could be easily removed with a small spatula. Pupae were weighed after tanning was complete and held for eclosion of adults.

To compare laboratory results with what might be found in the field, 10 soil samples $\left(250-\mathrm{cm}^{2}\right.$ cores, $15 \mathrm{~cm}$ in depth) were collected from a confinement dairy. Lots were prepared with sand similar to builder's sand $<3 \mathrm{wk}$ before samples were collected. Some samples were collected near mamure pats, but collection of visible amounts of raw manure was avoided. Samples were dried to determine moisture content. Sand content was determined by washing organic materials from dried samples, redrying, and weighing. Organic portion was determined by subtraction.

Data were analyzed with GLM procedures using the following nested factorial model: dependent variables $=$ moisture level + water/manure combination nested in moisture level. The Tukey studentized range test (SAS Institute 1985) was used for separation of means. Because of their relevance, only the separation of means between moisture levels within each water/manure combination have been shown in the tables. Unless otherwise stated, $P=0.05$.

\section{Results and Discussion}

Mean development time to the pupal stage ranged from 3.0 to $13.5 \mathrm{~d}$ (Table 2). As expected, there was no larval development in treatment 1 $(100 \%$ moisture from water) at any level. Inexplicably, there was no larval development in the $75 \%$ moisture from water and $25 \%$ moisture from mamure combination at the $100 \mathrm{ml}$ moisture level.
Nor was there larval development in the $75 \%$ moisture from water and $25 \%$ moisture from manure combination at the lowest and highest moisture levels. At the lowest moisture level, the nutrient quantities $(0.5 \mathrm{ml}$ per replication) apparently were insufficient, and at the highest level, high moisture levels (38.6\%) with comparatively low nutrient levels $(1.9 \%)$ probably contributed to larval mortality.

Mean duration of the pupal stage ranged from 3.0 to $11.5 \mathrm{~d}$ (Table 3 ). The time interval was greatest at the 2 lowest moisture levels, probably a result of the larvae developing at low nutritional levels. With the exception of moisture levels of 10 , $20,70,90$, and $100 \mathrm{ml}$, the duration range was between 3 and $4 \mathrm{~d}$ for most water/manure combinations.

Mean pupal weight ranged from 4.3 to $17.1 \mathrm{mg}$ (Table 4). Pupae tended to be larger with some degree of variation at moisture levels $\geq 50 \mathrm{ml}$, and larger with considerable uniformity in those same moisture levels when $100 \%$ of the moisture was from manure.

Mean number of pupae ranged from 1 to 53 (Table 5). The greatest number of pupae generally were produced in moisture levels $\geq 30 \mathrm{ml}$ and in water/manure combinations $3-5$. In 4 instances, the mean number of pupae was between 50 and 53 because of a counting error that occurred when larvae were applied to treatments.

Mean adult survival (based on original number of larvae) was $>50 \%$ in water/manure combinations $3-5$ at moisture levels $\geq 30 \mathrm{ml}$ (Table 6). In water/manure combination 2 , mean adult survival increased from $1.0 \%$ at the $20-\mathrm{ml}$ moisture level to $96.5 \%$ at the $70-\mathrm{ml}$ moisture level, then decreased to $3.0 \%$ at the $120-\mathrm{ml}$ moisture level. House fly survival was better than expected in most of the moisture/manure solids combinations used in this study.

Moisture content of field-collected samples from the confinement dairy was similar to laboratory moisture levels 10-40 (Table 7). However, manure solids in field-collected samples exceeded all levels tested in the laboratory. The highest proportion of manure solids tested in the laboratory was $7.3 \%$, but the lowest proportion of manure solids found in the field-collected samples was $47.7 \%$. Because of the high proportion of manure solids, the percentage of sand in all of the field-collected samples was lower than that of the laboratory samples.

Development of house fly larvae in the test substrates occurred in nearly all water/manure combinations containing manure solids. The shortest development time, $3 \mathrm{~d}$, occurred in water/manure combinations $2-5$ at the $70-\mathrm{ml}$ moisture level and in water/manure combinations $3-5$ at the $80-\mathrm{ml}$ moisture level (Table 2). This was $\approx 2 \mathrm{~d}$ shorter than estimates for larval development at comparable temperatures given by Larsen and Thomsen (1940) and Morgan (1986). 
Table 1. Composition of test substrates shown by moisture levels and water/manure combinations

\begin{tabular}{|c|c|c|c|c|c|c|}
\hline Water : manure ratio & Combination & Free water, $\mathrm{ml}$ & $\begin{array}{c}\text { Manure } \\
\text { moisture, } \mathrm{ml}\end{array}$ & $\begin{array}{c}\text { Manure } \\
\text { solids, ml }\end{array}$ & \% solids & \% moisture \\
\hline \multicolumn{7}{|c|}{ Level 10: } \\
\hline $100: 0$ & 1 & 10.0 & 0.0 & 0.0 & 0.00 & 4.76 \\
\hline $75: 25$ & 2 & 7.5 & 2.5 & 0.6 & 0.27 & 4.75 \\
\hline $50: 50$ & 3 & 5.0 & 5.0 & 1.2 & 0.54 & 4.74 \\
\hline $25: 75$ & 4 & 2.5 & 7.5 & 1.7 & 0.81 & 4.72 \\
\hline $0: 100$ & 5 & 0.0 & 10.0 & 2.3 & 1.08 & 4.71 \\
\hline \multicolumn{7}{|c|}{ Level 20: } \\
\hline $100: 0$ & 1 & 20.0 & 0.0 & 0.0 & 0.00 & 9.09 \\
\hline $75: 25$ & 2 & 15.0 & 5.0 & 1.2 & 0.52 & 9.04 \\
\hline $50: 50$ & 3 & 10.0 & 10.0 & 2.3 & 1.03 & 9.00 \\
\hline $25: 75$ & 4 & 5.0 & 15.0 & 3.5 & 1.54 & 8.95 \\
\hline $0: 100$ & 5 & 0.0 & 20.0 & 4.6 & 2.05 & 8.91 \\
\hline \multicolumn{7}{|c|}{ Level 30: } \\
\hline $100: 0$ & 1 & 30.0 & 0.0 & 0.0 & 0.00 & 13.04 \\
\hline $75: 25$ & 2 & 22.5 & 7.5 & 1.7 & 0.74 & 12.95 \\
\hline $50: 50$ & 3 & 15.0 & 15.0 & 3.5 & 1.48 & 12.85 \\
\hline $25: 75$ & 4 & 7.5 & 22.5 & 5.2 & 2.20 & 12.76 \\
\hline $0: 100$ & 5 & 0.0 & 30.0 & 6.9 & 2.91 & 12.66 \\
\hline \multicolumn{7}{|c|}{ Level 40: } \\
\hline $100: 0$ & 1 & 40.0 & 0.0 & 0.0 & 0.00 & 16.67 \\
\hline $75: 25$ & 2 & 30.0 & 10.0 & 2.3 & 0.95 & 16.51 \\
\hline $50: 50$ & 3 & 20.0 & 20.0 & 4.6 & 1.88 & 16.35 \\
\hline $25: 75$ & 4 & 10.0 & 30.0 & 6.9 & 2.79 & 16.20 \\
\hline $0: 100$ & 5 & 0.0 & 40.0 & 9.2 & 3.69 & 16.05 \\
\hline \multicolumn{7}{|c|}{ Level 50: } \\
\hline $100: 0$ & 1 & 50.0 & 0.0 & 0.0 & 0.00 & 20.00 \\
\hline $75: 25$ & 2 & 37.5 & 12.5 & 2.9 & 1.14 & 19.77 \\
\hline $50: 50$ & 3 & 25.0 & 25.0 & 5.8 & 2.25 & 19.55 \\
\hline $25: 75$ & 4 & 12.5 & 37.5 & 8.6 & 3.34 & 19.33 \\
\hline $0: 100$ & 5 & 0.0 & 50.0 & 11.5 & 4.40 & 19.12 \\
\hline \multicolumn{7}{|c|}{ Level 60: } \\
\hline 100:0 & 1 & 60.0 & 0.0 & 0.0 & 0.00 & 23.08 \\
\hline 75.25 & 2 & 45.0 & 15.0 & 3.5 & 1.31 & 22.78 \\
\hline $50: 50$ & 3 & 30.0 & 30.0 & 6.9 & 2.59 & 22.48 \\
\hline $25: 75$ & 4 & 15.0 & 45.0 & 10.4 & 3.83 & 22.19 \\
\hline $0: 100$ & 5 & 0.0 & 60.0 & 13.8 & 5.04 & 21.91 \\
\hline \multicolumn{7}{|c|}{ Level 70: } \\
\hline $100: 0$ & 1 & 70.0 & 0.0 & 0.0 & 0.00 & 25.93 \\
\hline $75: 25$ & 2 & 52.5 & 17.5 & 4.0 & 1.47 & 25.55 \\
\hline $50: 50$ & 3 & 35.0 & 35.0 & 8.1 & 2.90 & 25.18 \\
\hline $25: 75$ & 4 & 17.5 & 52.5 & 12.1 & 4.28 & 24.82 \\
\hline $0: 100$ & 5 & 0.0 & 70.0 & 16.1 & 5.63 & 24.47 \\
\hline \multicolumn{7}{|c|}{ Level 80: } \\
\hline $100: 0$ & 1 & 80.0 & 0.0 & 0.0 & 0.00 & 28.57 \\
\hline $75: 25$ & 2 & 60.0 & 20.0 & 4.6 & 1.62 & 28.11 \\
\hline $50: 50$ & 3 & 40.0 & 40.0 & 9.2 & 3.18 & 27,66 \\
\hline $25: 75$ & 4 & 20.0 & 60.0 & 13.8 & 4.70 & 27.23 \\
\hline $0: 100$ & 5 & 0.0 & 80.0 & 18.4 & 6.17 & 26.81 \\
\hline \multicolumn{7}{|c|}{ Level 90: } \\
\hline $100: 0$ & 1 & 90.0 & 0.0 & 0.0 & 0.00 & 31.04 \\
\hline $75: 25$ & 2 & 67.5 & 22.5 & 5.2 & 1.75 & 30.49 \\
\hline $50: 50$ & 3 & 45.0 & 45.0 & 10.4 & 3.45 & 29.97 \\
\hline $25: 75$ & 4 & 22.5 & 67.5 & 15.5 & 5.08 & 29.46 \\
\hline $0: 100$ & 5 & 0.0 & 90.0 & 20.7 & 6.66 & 28.97 \\
\hline \multicolumn{7}{|c|}{ Level 100: } \\
\hline $100: 0$ & 1 & 100.0 & 0.0 & 0.0 & 0.00 & 33.33 \\
\hline $75: 25$ & 2 & 75.0 & 25.0 & 5.8 & 1.88 & 32.71 \\
\hline $50: 50$ & 3 & 50.0 & 50.0 & 11.5 & 3.69 & 32.10 \\
\hline $25: 75$ & 4 & 25.0 & 75.0 & 17.3 & 5.44 & 31.52 \\
\hline $0: 100$ & 5 & 0.0 & 100.0 & 23.0 & 7.12 & 30.96 \\
\hline \multicolumn{7}{|c|}{ Level 110: } \\
\hline $100: 0$ & 1 & 110.0 & 0.0 & 0.0 & 0.00 & 35.48 \\
\hline $75: 25$ & 2 & 82.5 & 27.5 & 6.3 & 2.00 & 34.77 \\
\hline $50: 50$ & 3 & 55.0 & 55.0 & 12.7 & 3.92 & 34.09 \\
\hline $25: 75$ & 4 & 27.5 & 82.5 & 19.0 & 5.77 & 33.44 \\
\hline $0: 100$ & 5 & 0.0 & 110.0 & 25.3 & 7.55 & 32.81 \\
\hline
\end{tabular}


Table 1. Continued.

\begin{tabular}{ccccccc}
\hline \hline Wattr : manure ratio & Combination & Free water, $\mathrm{ml}$ & $\begin{array}{c}\text { Manure } \\
\text { moisture, } \mathrm{ml}\end{array}$ & $\begin{array}{c}\text { Manure } \\
\text { solids, } \mathrm{ml}\end{array}$ & \% solids & \% moisture \\
\hline & & \multicolumn{5}{c}{ Level 120: } \\
$100: 0$ & 1 & 120.0 & 0.0 & 0.0 & 0.00 & 37.50 \\
$75: 25$ & 2 & 90.0 & 30.0 & 6.9 & 2.11 & 36.71 \\
$50: 50$ & 3 & 60.0 & 60.0 & 13.8 & 4.13 & 35.95 \\
$25: 75$ & 4 & 30.0 & 90.0 & 20.7 & 6.08 & 35.22 \\
$0: 100$ & 5 & 0.0 & 120.0 & 27.6 & 7.94 & 34.52 \\
& & & Level 130: & & & \\
$100: 0$ & 1 & 130.0 & 0.0 & 0.0 & 0.00 & 39.39 \\
$75: 25$ & 2 & 97.5 & 32.5 & 7.5 & 2.22 & 38.52 \\
$50: 50$ & 3 & 65.0 & 65.0 & 15.0 & 4.33 & 37.69 \\
$25: 75$ & 4 & 32.5 & 97.5 & 22.4 & 6.36 & 36.89 \\
$0: 100$ & 5 & 0.0 & 130.0 & 29.9 & 8.31 & 36.12 \\
\hline
\end{tabular}

Percentage of sand + percentage manure solids + percentage moisture $=100 \%$. Moisture level $=$ moisture $(\mathrm{ml})$ per treatment replicate $=$ manure moisture + free water. Total sand $=272.2 \mathrm{~g}$ per treatment replicate.

Apparently, $0.5 \mathrm{ml}$ of manure solids was not enough to support the complete development of 50 house fly larvae, because dead larvae instead of pupae were found in the substrate in water/manure combination 2 at the $10-\mathrm{ml}$ moisture level (Table 1). The sand substrate in the 2 lowest water/ manure combinations was colored just slightly by the small amount of manure it contained. As fly larvae fed on the nutrients in the substrate, starting at the top and working downward, the sand was returned to its original white color. In these water/ manure combinations, larvae pupated throughout the medium, possibly near the location where feeding last occurred. There was a line in the substrate denoting where feeding had occurred above, and had not yet occurred below. Nutrients in water/manure combination 2 at the $10-\mathrm{ml}$ moisture level appeared to have been removed completely from the sand.

The 3- to 4-d pupal stadium compares well with the 4-d pupal stadium reported by Morgan (1986).

Table 2. Means of the larval stadia (d) of house flies reared in test substrates consisting of sand, manure solids, and moisture

\begin{tabular}{|c|c|c|c|c|c|}
\hline \multirow{2}{*}{$\begin{array}{c}\text { Mois- } \\
\text { ture } \\
\text { level, } \\
\mathrm{ml}\end{array}$} & \multicolumn{5}{|c|}{$\%$ manure moisture $(n)$} \\
\hline & 0 & 25 & 50 & 75 & 100 \\
\hline 10 & - & - & $13.5(3)_{\mathrm{a}}$ & $13.5(3) \mathrm{a}$ & $12.5(4) \mathrm{a}$ \\
\hline 20 & - & $11.5(2) \mathrm{a}$ & $11.8(2) \mathrm{b}$ & $10.0(3) \mathrm{b}$ & $8.5(4) \mathrm{b}$ \\
\hline 30 & - & $7.0(4) \mathrm{b}$ & $8.0\langle 4\rangle \mathrm{c}$ & $6.0(4) \mathrm{d}$ & $5.0(4) \mathrm{e}$ \\
\hline 40 & $\ldots$ & $5.0(4) \mathrm{d}$ & $5.0(4) \mathrm{e}$ & $4.0(4) \mathrm{f}$ & $4.0(4) \mathrm{f}$ \\
\hline 50 & - & $6.0(4) \mathrm{c}$ & $6.0(4) \mathrm{d}$ & $5.0(4) \mathrm{e}$ & $5.0(4) \mathrm{e}$ \\
\hline 60 & $\cdots$. & $5.0(4) \mathrm{d}$ & $5.0(4) \mathrm{e}$ & $5.0(4) \mathrm{e}$ & $5.0(4) \mathrm{e}$ \\
\hline 70 & - & $3.0(4) \mathrm{e}$ & $3.0(4) \mathrm{g}$ & $3.0(4) \mathrm{g}$ & $3.0(4) \mathrm{g}$ \\
\hline 80 & - & $6.0(4) \mathrm{c}$ & $3.0(4) \mathrm{g}$ & $3.0(4) \mathrm{g}$ & $3.0(4) \mathrm{g}$ \\
\hline 90 & - & $5.0(4) \mathrm{d}$ & $4.0(4) f$ & $4.0(4) \mathrm{f}$ & $4.0(4) \mathrm{f}$ \\
\hline 100 & - & - & $5.0(4) \mathrm{e}$ & $6.0(4) \mathrm{d}$ & $6.0(4) \mathrm{d}$ \\
\hline 110 & - & $6.0(4) \mathrm{e}$ & $6.0(4) \mathrm{d}$ & $6.0(4) \mathrm{d}$ & $6.0(4) \mathrm{d}$ \\
\hline 120 & - & $5.0(2) \mathrm{d}$ & $5.0(4) \mathrm{e}$ & $7.0(4) \mathrm{c}$ & $7.0(4) c$ \\
\hline 130 & - & - & $5.0(4) \mathrm{e}$ & $5.0(4) \mathrm{e}$ & $5.0(4) \mathrm{e}$ \\
\hline
\end{tabular}

Means in the same column followed by the same letter are not significantly different ( $P=0.05$, Tukey studentized range test [SAS Institute 1985]). Moisture level $=$ moisture $(\mathrm{ml})$ per treatment replicate $=$ manure moisture + free water.
The shortest time from 1st instar to adult was 6-7 $\mathrm{d}$, which was the shortest development time reported by Larsen and Thomsen (1940). Pupal weights generally were close to the 13- to $14-\mathrm{mg}$ size reported by Morgan (1986) and Hogsette (1992).

The survival rates in many cases compared favorably with the $73-80 \%$ reported by Morgan (1986) (Table 6). Survival in the 10- and $20-\mathrm{ml}$ moisture ranges was reduced most likely by nutrition. However, a reduction in survival in water/manure combination 2 at the $120-$ and $130-\mathrm{ml}$ levels was probably the result of nutrient dilution and relatively high moisture levels.

Confining the sand in cups causes it to hold more water than it would under most field conditions. A $200-\mathrm{cm}^{3}$ cup of builder's sand becomes completely saturated with the addition of $83-85 \mathrm{ml}$ of water. Thus, in water/manure combinations having $\geq 90 \mathrm{ml}$ of moisture, water puddled on the sand surface, particularly in treatments $1-3$, until it was

Table 3. Means of the pupal stadia (d) of house flies reared in test substrates consisting of sand, manure solids, and moisture

\begin{tabular}{rccccc}
\hline \hline $\begin{array}{c}\text { Mois- } \\
\text { ture } \\
\text { level, } \\
\text { ml }\end{array}$ & 0 & 25 & 50 & 75 & 100 \\
\hline 10 & - & - & $8.0(3) \mathrm{a}$ & $8.0(3) \mathrm{b}$ & $8.5(4) \mathrm{b}$ \\
20 & - & $8.5(2) \mathrm{a}$ & $7.3(2) \mathrm{a}$ & $9.0(3) \mathrm{a}$ & $11.5(4) \mathrm{a}$ \\
30 & - & $4.5(4) \mathrm{b}$ & $4.5(4) \mathrm{cd}$ & $3.0(4) \mathrm{f}$ & $4.0(4) \mathrm{e}$ \\
40 & - & $3.0(4) \mathrm{c}$ & $4.0(4) \mathrm{d}$ & $4.0(4) \mathrm{e}$ & $4.0(4) \mathrm{e}$ \\
50 & - & $3.0(4) \mathrm{c}$ & $3.0(4) \mathrm{e}$ & $3.0(4) \mathrm{f}$ & $3.0(4) \mathrm{f}$ \\
60 & - & $4.0(4) \mathrm{bc}$ & $4.0(4) \mathrm{d}$ & $4.0(4) \mathrm{e}$ & $4.0(4) \mathrm{e}$ \\
70 & - & $5.0(4) \mathrm{b}$ & $5.0(4) \mathrm{c}$ & $5.0(4) \mathrm{d}$ & $5.0(4) \mathrm{d}$ \\
80 & - & $4.0(4) \mathrm{bc}$ & $4.0(4) \mathrm{d}$ & $4.0(4) \mathrm{e}$ & $4.0(4) \mathrm{e}$ \\
90 & - & $5.0(4) \mathrm{b}$ & $5.0(4) \mathrm{c}$ & $5.0(4) \mathrm{d}$ & $5.0(4) \mathrm{d}$ \\
100 & - & $0.0(4) \mathrm{d}$ & $6.0(4) \mathrm{b}$ & $7.0(4) \mathrm{c}$ & $7.0(4) \mathrm{c}$ \\
110 & - & $3.0(4) \mathrm{c}$ & $3.0(4) \mathrm{e}$ & $3.0(4) \mathrm{f}$ & $3.0(4) \mathrm{f}$ \\
120 & - & $4.0(2) \mathrm{bc}$ & $4.0(4) \mathrm{d}$ & $3.0(4) \mathrm{f}$ & $4.0(4) \mathrm{e}$ \\
130 & - & - & $3.0(4) \mathrm{e}$ & $4.0(4) \mathrm{e}$ & $5.0(4) \mathrm{d}$ \\
\hline
\end{tabular}

Means in the same column followed by the same letter are not significantly different $(P=0.05$, Tukey studentized range test [SAS Institute 1985]). Moisture level $=$ moisture $(\mathrm{ml})$ per treatment replicate $=$ manure moisture + free water. 
Table 4. Means of the pupal weight (mg) of house flies reared in test substrates $(n=$ replicates) consisting of sand, manure solids, and moisture

\begin{tabular}{cccccr}
\hline \hline $\begin{array}{c}\text { Mois- } \\
\text { ture } \\
\text { level, }\end{array}$ & 0 & 25 & 50 & 75 & 100 \\
$\mathrm{ml}$ & & & & & \\
\hline 10 & - & - & $4.9(3) \mathrm{g}$ & $4.3(4) \mathrm{e}$ & $5.0(4) \mathrm{a}$ \\
20 & - & $5.8(2) \mathrm{d}$ & $4.3(2) \mathrm{g}$ & $4.5(4) \mathrm{e}$ & $6.3(4) \mathrm{c}$ \\
30 & - & $5.6(4) \mathrm{d}$ & $9.3(4) \mathrm{f}$ & $7.9(4) \mathrm{d}$ & $10.7(4) \mathrm{b}$ \\
40 & - & $5.3(4) \mathrm{d}$ & $6.1(4) \mathrm{g}$ & $9.4(4) \mathrm{d}$ & $9.8(4) \mathrm{b}$ \\
50 & - & $14.0(4) \mathrm{ab}$ & $10.7(4) \mathrm{ef}$ & $9.4(4) \mathrm{d}$ & $14.6(4) \mathrm{a}$ \\
60 & - & $9.4(4) \mathrm{c}$ & $12.4(4) \mathrm{de}$ & $14.6(4) \mathrm{ab}$ & $14.9(4) \mathrm{a}$ \\
70 & - & $10.6(4) \mathrm{bc}$ & $12.0(4) \mathrm{de}$ & $13.7(4) \mathrm{bc}$ & $14.7(4) \mathrm{a}$ \\
80 & - & $10.9(4) \mathrm{bc}$ & $13.2(4) \mathrm{bc}$ & $13.2(4) \mathrm{c}$ & $14.6(4) \mathrm{a}$ \\
90 & - & $11.1(4) \mathrm{bc}$ & $16.1(4) \mathrm{a}$ & $15.2(4) \mathrm{ab}$ & $14.5(4) \mathrm{a}$ \\
100 & - & $0.0(4) \mathrm{e}$ & $15.1(4) \mathrm{ab}$ & $15.1(4) \mathrm{ab}$ & $15.9(4) \mathrm{a}$ \\
110 & - & $15.0(4) \mathrm{a}$ & $12.5(4) \mathrm{cd}$ & $14.0(4) \mathrm{bc}$ & $14.5(4) \mathrm{a}$ \\
120 & - & $17.1(2) \mathrm{a}$ & $1.5 .2(4) \mathrm{ab}$ & $16.4(4) \mathrm{a}$ & $16.0(4) \mathrm{a}$ \\
130 & - & - & $14.6(4) \mathrm{ab}$ & $14.6(4) \mathrm{ab}$ & $14.6(4) \mathrm{a}$ \\
\hline
\end{tabular}

Means in the same column followed by the same letter are not significantly different $(P=0.05$, Tukey studentized range test [SAS lnstitute 1985]). Moisture level $=$ moisture $(\mathrm{ml})$ per treatment replicate $=$ manure moisture + free water.

lost by evaporation in the growth chamber. The higher moisture levels did not prevent fly development except in water/manure combination 2 at the 120- and $130-\mathrm{ml}$ levels where most of the moisture, 36.8 and $38.6 \%$, respectively, was not associated with manure, i.e., moisture was added as free water. Moisture in the field-collected samples did not exceed $31.3 \%$.

In Nebraska feedlots, fly larvae are most numerous in habitats that were not routinely trod upon by cattle (Meyer and Petersen 1983, Skoda et al. 1993). Soil substrates apparently pack tightly enough under the weight of the cattle to kill fly larvae developing within. Enabling feedlot cattle to walk on manure-laden soil containing fly larvae has been recommended as a fly control technique

Table 5. Mean number of house fly pupae in test substrates consisting of sand, manure solids, and moisture

\begin{tabular}{|c|c|c|c|c|c|}
\hline \multirow{2}{*}{$\begin{array}{l}\text { Mois- } \\
\text { ture } \\
\text { level, } \\
\mathrm{ml}\end{array}$} & \multicolumn{5}{|c|}{$\%$ manure moisture $(n)$} \\
\hline & 0 & 25 & 50 & 75 & 100 \\
\hline 10 & - & - & $4.3(4) \mathrm{d}$ & $2.3(4) \mathrm{b}$ & $8.0(4) \mathrm{b}$ \\
\hline 20 & - & $0.8(4) \mathrm{cd}$ & $3.5(4) \mathrm{d}$ & $7.8(4) \mathrm{b}$ & $19.3(4) \mathrm{b}$ \\
\hline 30 & - & $14.3(4) c$ & $32.3(4) \mathrm{c}$ & $44.5(4) a$ & $47.3(4) \mathrm{a}$ \\
\hline 40 & - & $12.0(4) \mathrm{cd}$ & $44.0(4) \mathrm{ab}$ & $45.5(4) \mathrm{a}$ & $46.5(4)_{\mathrm{a}}$ \\
\hline 50 & - & $36.8(4) \mathrm{b}$ & $40.5(4) \mathrm{ab}$ & $42.8(4) \mathrm{a}$ & $39.0(4) \mathrm{a}$ \\
\hline 60 & - & $44.8(4) \mathrm{ab}$ & $42.0(4) \mathrm{ab}$ & $39.8(4) a$ & $41.8(4) \mathrm{a}$ \\
\hline 70 & - & $52.5(4) \mathrm{a}$ & $50.5(4) \mathrm{a}$ & $43.5(4)_{\mathrm{a}}$ & $45.3(4) \mathrm{a}$ \\
\hline 80 & - & $39.5(4) \mathrm{ab}$ & $33.3(4) b c$ & $38.5(4) a$ & $39.5(4) a$ \\
\hline 90 & - & $41.3(4) \mathrm{ab}$ & $46.8(4) \mathrm{ab}$ & $47.5(4) \mathrm{a}$ & $46.8(4) \mathrm{a}$ \\
\hline 100 & - & $0.0(4) \mathrm{d}$ & $49.3(4) \mathrm{ab}$ & $51.0(4) \mathrm{a}$ & $50.0(3) \mathrm{a}$ \\
\hline 110 & - & $43.5(4) \mathrm{ab}$ & $41.0(4) \mathrm{ab}$ & $40.5(4) \mathrm{a}$ & $45.5(4) \mathrm{a}$ \\
\hline 120 & - & $1.5(4) \mathrm{cd}$ & $36.5(4) a b$ & $43.3(4) \mathrm{a}$ & $48.3(4) \mathrm{a}$ \\
\hline 130 & - & - & $49.0(4) \mathrm{ab}$ & $48.8(4) \mathrm{a}$ & $48.3(4) \mathrm{a}$ \\
\hline
\end{tabular}

Means in the same column followed by the same letter are not significantly different $(P=0.05$, Tukey studentized range test [SAS Institute 1985]). Moisture level $=$ moisture $(\mathrm{ml})$ per treatment replicate $=$ manure moisture + free water.
Table 6. Mean percentage adult survival of house flies reared in test substrates consisting of sand, manure solids, and moisture

\begin{tabular}{|c|c|c|c|c|c|}
\hline \multirow{2}{*}{$\begin{array}{c}\text { Mois- } \\
\text { ture } \\
\text { level, } \\
\text { ml }\end{array}$} & \multicolumn{5}{|c|}{$\%$ manure moisture $(n)$} \\
\hline & 0 & 25 & 50 & 75 & 100 \\
\hline 10 & - & - & $7.5(4) c$ & $4.5(4) b$ & $13.5(4) c$ \\
\hline 20 & - & $1.0(4) \mathrm{c}$ & $4.0(4) c$ & $9.5(4) \mathrm{b}$ & $29.5(4) b c$ \\
\hline 30 & - & $24.5(4) c$ & $54.5(4) \mathrm{b}$ & $83.5(4) \mathrm{a}$ & $9(0.0)(4) \mathrm{a}$ \\
\hline 40 & - & $20.0(4) \mathrm{c}$ & $78.5(4) \mathrm{ab}$ & $86.5(4) \mathrm{a}$ & $89.5(4)_{a}$ \\
\hline 50 & - & $66.5(4) \mathrm{b}$ & $76.5(4) \mathrm{ab})$ & $79.5(4)_{a}$ & $71.0(4)_{\mathrm{a}}$ \\
\hline 60 & - & $75.0(4) \mathrm{ab}$ & $75.0(4) \mathrm{ab}$ & $67.5(4) \mathrm{a}$ & $71.5(4) \mathrm{a}$ \\
\hline 70 & - & $96.5(4)_{\mathrm{a}}$ & $95.5(4)_{\mathrm{i}}$ & $81.5(4)_{\mathrm{a}}$ & $8.4 .5(4)_{\mathrm{a} 1}$ \\
\hline 80 & - & $76.5(4) \mathrm{ab}$ & $63.5(4) a b$ & $72.5(4)_{\mathrm{a}}$ & $75.0)(4)_{\mathrm{a}}$ \\
\hline 90 & - & $80.0(4) \mathrm{ab}$ & $89.5(4)$ 丸 & $89.6(4)_{\mathrm{a}}$ & $93.5(4) \mathrm{a}$ \\
\hline 100 & - & $0.0(4) \mathrm{c}$ & $92.5(4) \mathrm{a}$ & $96.5(4)_{\mathrm{a}}$ & $66.0(4) \mathrm{ab}$ \\
\hline 110 & - & $78.0(4) \mathrm{ab}$ & $74.1)(4) a b$ & $75.0(4)_{\mathrm{a}}$ & $79.5(4) \mathrm{a}$ \\
\hline 120 & - & $3.0(4) c$ & $70.0(4) \mathrm{ab}$ & $83.0(4) a$ & $42.5(4) \mathrm{a}$ \\
\hline 130 & - & - & $94.0(4) \mathrm{a}$ & $95.5(4)_{\mathrm{a}}$ & $94 .(1)(4) \mathrm{a}$ \\
\hline
\end{tabular}

Means in the same column followed by the same letter are not significantly different ( $P=0.05$, Tukey studentized range test [SAS Institute 1985]). Moisture level $=$ moisture (ml) per treatment replicate $=$ manure moisture + frees water.

(McNeal and Campbell 1981). However, Skoda et al. (1993) consistently found small numbers of house fly larvae in general lot areas used daily by cattle.

Large concentrations of fly larvae on California dairies were found in moist areas near water troughs and in stacked manure (Meyer and Schultz 1990). But, moderate numbers of fly larvae were also recovered from lots occupied by animals.

The most revealing facts ascertained from this study are that house flies can develop in $200 \mathrm{~cm}^{3}$ of coarse sand containing just $1 \mathrm{ml}(0.47 \%)$ of manure solids and $10 \mathrm{ml}(4.74 \%)$ of moisture. Development is slow (21.5 d from lst instar to adult), adult survival is low $(7.5 \%)$, but successful development can occur. The potential problems for fly control are dependent on how much fly development actually occurs in manure-soil substrates in pastures and confinement lots. More studies are needed to learn how fly larvae use nutrient rich soil substrates in conjunction with associated physical and microclimatic variables, e.g., soil compaction, moisture, and temperature. Until we can map the areas of larval fly development on pasture and

Table 7. Volumetric analysis of soil samples collected from prepared sand lots being used by eattle on a confinement dairy

\begin{tabular}{cccc}
\hline \hline Sample no. & \% moisture & \% sand & $\%$ manure solids \\
\hline 1 & 8.5 & 43.9 & 47.7 \\
2 & 17.7 & 25.0 & 57.3 \\
3 & 13.2 & 21.0 & 65.8 \\
4 & 6.9 & 14.1 & 79.1 \\
5 & 7.6 & 11.1 & 81.3 \\
6 & 11.2 & 7.0 & 81.9 \\
7 & 2.8 & 7.4 & 89.8 \\
8 & 6.8 & 3.9 & 89.3 \\
9 & 6.3 & 3.5 & 90.2 \\
10 & 5.1 & 2.0 & 93.0 \\
\hline
\end{tabular}


confinement cattle facilities and predict the contribution that these areas make to the total adult fly populations on the facilities, fly management will remain an elusive proposition.

\section{Acknowledgment}

I thank G. Langley and A. Lee (USDA-ARS) Gainesville, FL, for their technical assistance.

\section{References Cited}

Bishopp, F. C., W. E. Dove, and D. C. Parman. 1915. Notes on certain points of economic importance in the biology of the house fly. J. Econ. Entomol. 8: 54-71.

Bryant, E. H., and A. E. Hall. 1975. The role of medium conditioning in the population dynamics of the housefly. Res. Popul. Ecol. 16: 188-197.

Hewitt, C. G. 1914. The house fly. Cambridge University Press, Cambridge.

Hogsette, J. A. 1992. New diets for production of house flies and stable flies (Diptera: Muscidae) in the laboratory. J. Econ. Entomol. 85: 2291-2294.

Hogsette, J. A., and F. Washington. 1995. Quantitative mass production of Hydrotaea aenescens (Wiedemann) (Diptera: Muscidae). J. Econ. Entomol. 88: $1238-1242$.

James, M. T. 1947. The flies that cause myiasis in man. U.S. Dep. Agric. Misc. Publ. 631.

Keiding, J. 1976. The house-fly-biology and control. WHO Rep. 76.650.

Larsen E. B., and M. Thomsen. 1940. The influence of temperature on the development of some species of Diptera. Vidensk. Medd. Dan. Naturhist. Foren. 104: 1-75.

MeNeal, C. D., Jr., and J. B. Campbell. 1981. Insect pest management in Nebraska feedlots and dairies: a pilot integrated pest management project. Coop. Ext. Serv. Inst. Agric. Nat. Resour. Rep. 10.

Mellor, J.E.M. 1919. Observations on the habits of certain flies, especially of those breeding in manure. Ann. Appl. Biol. 6: 53-88.
Meyer, J. A., and J. J. Petersen. 1983. Characterization and seasonal distribution of breeding sites of stable flies and house flies on eastern Nebraska feedlots and dairies. J. Econ. Entomol. 76: 103-108.

Meyer, J. A., und T. A. Shmtz. 1990. Stable Hy and house fly breeding sites on dairies. Calif. Agric. 44: 28-29.

Morgan, P. B. 1986. Mass culturing microhymenopteran pupal parasites (Hymenoptera: Pteromalidae) of filth breeding flies, pp. 77-87. In R. S. Patterson and D. A. Rutz [eds.], Biological control of muscoid flies. Entomol. Soc. Am. Misc. Publ. 61.

Newstead, R. 1908. On the habits, life-cycle and breeding places of the common house-fly (Musca domestica, Linn.). Ann. Trop. Med. Parasitol. 1: 507-. 520.

Patterson, R. S. 1981. Importance of monitoring house fly and stable fly immature and adult populations in IPM programs using biocontrol, pp. 95-102. In Status of Biological Control of Filth Flies, Proceedings of a Workshop, USDAVScience and Education Administration, Institute of Food and Agricultural Sciences. University of Florida, Gainesville.

Rabari, P. M., and R. C. Patel. 1977. Breeding of housefly Musca domestica $\mathrm{L}$. in different media in rural areas. Indian J. Entomol. 39: 186-188.

Robinson, J., and M. L. Luff. 1976. The sheep headfly, Hydrotaea irritans (Fall.) (Diptera: Muscidae): Larval habitat and immature stages. Bull. Entomol. Res. 65: 579-586.

Saceà, G. 1964. Comparative bionomics in the genus Musca. Annu. Rev. Entomol. 9: 341-358.

SAS Institute. 1985. SAS user's guide: statistics. SAS Institute, Cary, NC.

Skoda, S. R., G. D. Thomas, und J. B. Camploell. 1993. Abundance of the immature stages of the: house fly (Diptera: Muscidae) from five areas in beef cattle feedlot pens. J. Econ. Entomol. 86: 455-461.

Thomas, G. D., and S. R. Skoda [eds.]. 1993. Rural flies in the urban environment. North Central Regional Research Publication 335, University of Nebraska, Lincoln.

Received for publication 7 June 1995; accepted 19 March 1996. 